\title{
GMR
}

\section{Three-step method for the amplification of the coxsackievirus A10 genome}

\author{
A.Y. Wang ${ }^{1,2 *}$, C.Y. Mu ${ }^{1,3 *}$, C. Chen ${ }^{3}$, Z. Li ${ }^{1}$ and Y.H. Xu' \\ ${ }^{1}$ Department of Microbiology, Anhui Medical University, Hefei, Anhui Province, \\ China \\ ${ }^{2}$ Department of Clinical Laboratory, First Affiliated Hospital of Anhui Medical University, \\ Hefei, Anhui Province, China \\ ${ }^{3}$ Department of Clinical Laboratory, Huaibei Miner's General Hospital, Huaibei, \\ Anhui Province, China \\ *These authors contributed equally to this study. \\ Corresponding authors: Z. Li / Y.H. Xu \\ E-mail: stronglz110732@163.com / blackpippy@163.com \\ Genet. Mol. Res. 15 (1): gmr.15017513 \\ Received August 25, 2015 \\ Accepted November 5, 2015 \\ Published February 19, 2016 \\ DOI http://dx.doi.org/10.4238/gmr.15017513
}

ABSTRACT. The whole-genome sequencing of coxsackievirus (CV)-A10 does not follow a conventional experimental protocol. To fully understand the genetic variation and evolution of CV-A10, complete genome amplification is necessary. Most previous studies have concentrated on partial sequences of the CV-A10 genome, such as the VP1 gene. The few studies that have investigated CV-A10 at the genomic level have reported only two complete genome sequences to GenBank. The basic fault may be attributed to the regional nature of the genetics and evolution of CVA10 and to the lack of laboratory procedures for obtaining the genomes. In this study, we present a robust "three-step" protocol performed with A105UF/A820, EVP4/A6141, and A4879/A1005R for the full-length genome amplification of CV-A10. The results revealed that the method is able to accurately and reproducibly amplify three fragments with overlaps of the full-length genome of eight CV-A10 strains. Compared with other methods, this assay is both quick and specific. In addition, the three-step 
protocol could be capable of amplifying the full-length genomes of CVA10 strains isolated from different countries and regions. The specific three-step protocol may be particularly useful for investigating samples co-infected with CV-A10 and other viruses.

Key words: CV-A10; Three-step protocol; Full-length genome amplification

\section{INTRODUCTION}

Hand, foot, and mouth disease (HFMD) is a common childhood disorder characterized by vesicular rashes on the palms, soles, and buttocks, and by oropharyngeal ulcers. In rare cases, patients also develop neurological complications, such as encephalomyelitis and aseptic meningitis (McMinn, 2002; Shah et al., 2003; Pérez-Vélez et al., 2007). Several large epidemics of HFMD have been reported in the Asia Pacific region, particularly in Southeast Asia, since 1997 (Lu et al., 2012). Previous studies have shown that outbreaks of HFMD are mainly caused by human enterovirus 71 (EV-71), CV-A6, and CV-A16 (Chang et al., 1999; Yan et al., 2001; Lin et al., 2003; Osterback et al., 2009; Zhang et al., 2009; Zhang et al., 2010). In addition, outbreaks or sporadic cases may also be caused by other human enteroviruses, such as CV-A10. In recent years, CV-A10-related HFMD has been associated with increasingly common but sporadic cases of HFMD, and by global outbreak events (Blomqvist et al., 2010; Wu et al., 2010; Yu and Song, 2010; Davia et al., 2011; Lu et al., 2012; Mirand et al., 2012; He et al., 2013). All of these studies strongly demonstrated that CV-A10 infections are an important cause of HFMD. Therefore, it is important to investigate the genetic variation and evolution of CV-A10 (Figure 1) that takes place during HFMD epidemics. Most previous studies have focused on partial sequences, such as the VP1 gene, owing to the absence of an efficient method for obtaining the full-length CV-A10 genome. The aim of this study was to develop a "three-step" amplification method for sequencing the CV-A10 genome.

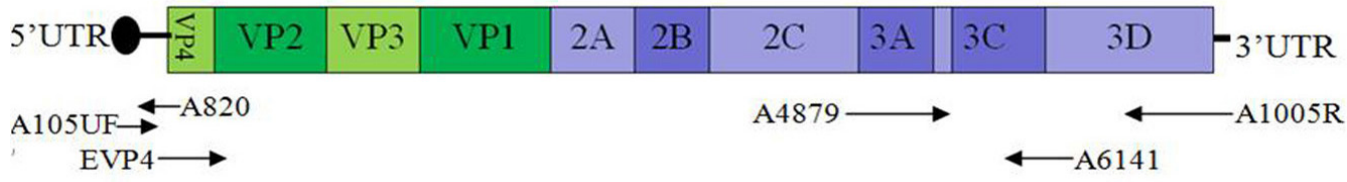

Figure 1. Schematic representation of the coxsackievirus A10 (CV-A10) genome. The genome comprises a polyadenylated positive-strand RNA sequence that is covalently linked to a small VPg (viral protein genome-linked) at the $5^{\prime}$ terminus. The single open-reading frame is flanked by two untranslated regions (5'UTR and 3'UTR). The position and direction of the primers used are indicated with bold arrows.

\section{MATERIAL AND METHODS}

\section{Viruses}

CV-A10 strains were isolated, identified, and transported to the Fuyang City Center for Disease Control and Prevention. All isolates were cultured using RD cells (a cell line derived from human rhabdomyosarcoma). According to the manufacturer instructions, the culture flasks were 
freeze-thawed once before the culture liquid was collected. The cellular debris was removed by low-speed centrifugation (4000 g, $10 \mathrm{~min}$ ). Subsequently, CV-A10 was concentrated using Amicon Ultra-15 (Millipore, Bedford, MA, USA) filtration tubes (100 kDa), and the virus-containing retentate was stored at $-80^{\circ} \mathrm{C}$.

\section{Purification of viral RNA}

The concentrated CV-A10 preparation was extracted and purified using an SV total RNA isolation kit (Promega, Beijing, China). The products were stored at $-80^{\circ} \mathrm{C}$ until required.

\section{Three-step reverse transcription polymerase chain reaction (RT-PCR) and six-step PCR}

Reverse transcription was performed using SuperScript ${ }^{\mathrm{TM}}$ Reverse Transcriptase (Invitrogen, Shanghai, China), and the products were stored at $-80^{\circ} \mathrm{C}$ until required. According to the manufacturer instructions, the complementary DNA (cDNA) was synthesized using oligo$\mathrm{dT}$ at $42^{\circ} \mathrm{C}$ for $50 \mathrm{~min}$. The cDNA was used directly for full-length PCR amplification or stored at $-80^{\circ} \mathrm{C}$.

Three-step PCR was successively performed with A105UF/A820, EVP4/A6141, and A4879/A1005R (Table 1) using PrimeSTAR GXL DNA Polymerase (TaKaRa, Dalian, China). The PCR regimen was as follows: 1) 35 cycles of $98^{\circ} \mathrm{C}$ for $10 \mathrm{~s}, 55^{\circ} \mathrm{C}$ for $15 \mathrm{~s}$, and $68^{\circ} \mathrm{C}$ for $35 \mathrm{~s}$; 2) 35 cycles of $98^{\circ} \mathrm{C}$ for $10 \mathrm{~s}, 63^{\circ} \mathrm{C}$ for $15 \mathrm{~s}$, and $68^{\circ} \mathrm{C}$ for $4 \mathrm{~min}$; and 3) 35 cycles of $98^{\circ} \mathrm{C}$ for $10 \mathrm{~s}, 55^{\circ} \mathrm{C}$ for $15 \mathrm{~s}$, and $68^{\circ} \mathrm{C}$ for $1 \mathrm{~min}$ and $20 \mathrm{~s}$. The products of eight CV-A10 strains were then subjected to $1.2 \%$ agarose gel electrophoresis (Figure 2). The A105UF, A1005R, and EVP4 primers were those described by Hu et al. (2011) and Ishiko et al. (2002). The A820, A6141, and A4879 primers were designed in reference to the Kowalik strain (AY421767) and the CV-A10/SD/CHN/09 strain (HQ728262), and synthesized by Invitrogen Co. (Invitrogen, Shanghai, China). The primers for the three-step protocol were used according to Figure 1.

Table 1. Primers for the amplification of coxsackievirus $A 10$ (CV-A10) using the three-step methoda.

\begin{tabular}{l|l|c|c}
\hline Primer & Sequence $\left(5^{\prime} \rightarrow 3^{\prime}\right)$ & Nucleotide position & Size of product (bp) \\
\hline A105UF & TTAAAACAGCCTGTGGGTTG & $1-20$ & 817 \\
\hline A820 & TTGTAGATCCTCCAGTGGC & $799-817$ or $820-802$ & \\
\hline EVP4 & CTACTTTGGGTGTCCGTGTT & $548-567$ or $551-570$ & \\
\hline A6141 & GTGGAGAACATTGCCCACAT & $6141-6122$ or $6144-6125$ & 5594 \\
\hline A4879 & AAGCTCTGCTCAGAGAACAACAC & $4879-4901$ or $4882-4904$ & 2488 \\
\hline
\end{tabular}

a5'UTR, EVP4, and A1005R refer to Ishiko et al. (2002) and Hu et al. (2011), and nucleotide positions were based on the genomes of the CV-A10-Kowalik strain (AY421767) or the CV-A10/SD/CHN/09 (HQ728262) strain.

The six-step PCR was carried out with six pairs of primers (Table 2) for the amplification of the whole-genome sequences of the CV-A10 strains (Hu et al., 2011). The six-step PCR was performed according to the following regimen: a total of 35 cycles of $30 \mathrm{~s}$ at $95^{\circ} \mathrm{C}, 30 \mathrm{~s}$ at $55^{\circ} \mathrm{C}$, and 0.5 to $3 \mathrm{~min}$ at $72^{\circ} \mathrm{C}$. The PCR products were visualized on $1.2 \%$ agarose gel (Figure 3 ) and compared with the gel shown in Figure 2. 


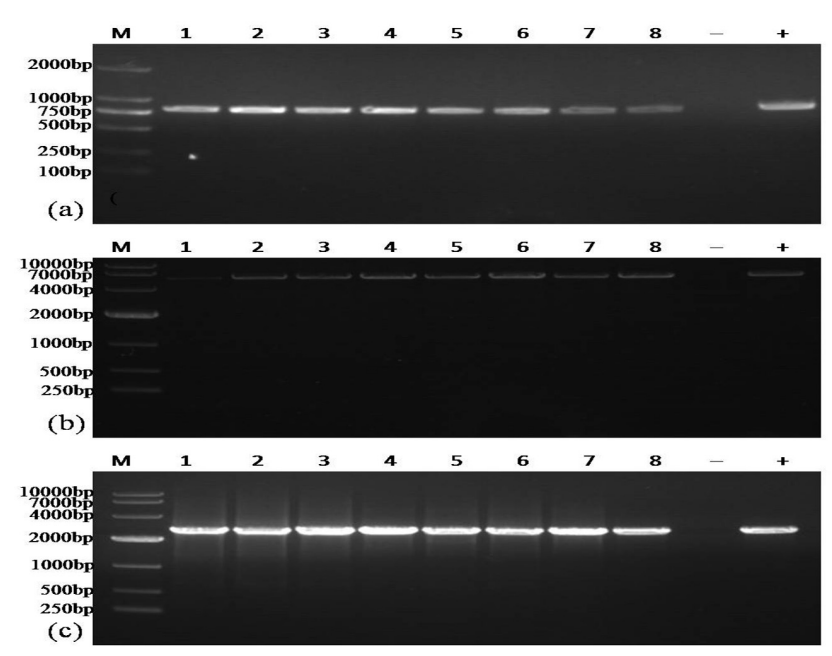

Figure 2. Agarose gel electrophoresis of the amplicons of A105UF/A820-PCR (a), EVP4/A6141-PCR (b), and A4879/ A1005R-PCR (c) of eight different coxsackievirus A10 (CV-A10) strains (PCR is polymerase chain reaction). The FY01 to FY08 strains are represented in lanes 1 to 8 , respectively, and the negative and positive controls are labeled with "-" and "+". The DL2000 DNA marker was used in a (TaKaRa, Dalian, China), and the DL10000 DNA Marker was used in $\mathbf{b}$ and $\mathbf{c}$ (TaKaRa, Dalian).

Table 2. Primers for the genome amplification of coxsackievirus $A 10$ (CV-A10) using the six-step method ${ }^{b}$.

\begin{tabular}{l|l|c|l|r}
\hline Primer & Region & Position & Sequence $\left(5^{\prime} \rightarrow 3^{\prime}\right)$ & Size of product (bp) \\
\hline A105UF & 5'UTR & $1-20$ & TTAAAACAGCCTGTGGGTTG & 488 \\
\hline A105UR & 5'UTR & $488-469$ & CAGTTAGGATTAGCCGCATT & 1671 \\
\hline A1001F & 5'UTR & $393-414$ & GGCAACCCATGGGACGCTCTAA & \\
\hline A1001R & VP3 & $2063-2040$ & CTTTAACGAGCCTGACCATTGTGT & 2077 \\
\hline A1002F & VP3 & $1732-1754$ & GAGCTCAGGCCCGGGACTAATCA & 1732 \\
\hline A1002R & 2B & $3808-3794$ & TTGATATAGTCAGACACTC & 1787 \\
\hline A1003F & 2B & $3720-3742$ & CCTCGTGGGGTTTGCTGATGTGA & \\
\hline A1003F & 3C & $5428-5451$ & GTCGGTCTGCGCTTGTCTGATGTT & \\
\hline A1004F & 2A & $5270-5293$ & TTGCGGTTGTGTCCCTTGTGTATG & \\
\hline A1005F & 3D & $7056-7033$ & GGTTGCGTTCTCCCAGGTGACTTC & \\
\hline A1005R & 3'UTR & $6957-6974$ & CTAAACATGGTGGCCTACGGAGAT & \\
\hline
\end{tabular}

${ }^{\mathrm{b}}$ Table 2 refers to $\mathrm{Hu}$ et al. (2011), and nucleotide positions were based on the genome of the CV-A10/SD/CHN/09 (HQ728262) strain.

\section{Verification of the three-step assay}

To verify the results of the three-step protocol, all the amplicons produced using the A105UF/A820, EVP4/A6141, and A4879/A1005R primers were sequenced and assembled by Sangon Co. (Sangon, Shanghai, China). The near full-length genome sequences of eight CV-A10 strains were registered at GenBank.

\section{RESULTS}

\section{Whole-genome amplification using the three-step protocol}

The amplification of the whole-genome sequences of CV-A10 was conducted using primers 
in accordance with Figure 1. Using the three-step protocol proposed, the complete genomes of eight CV-A10 strains were obtained using three fragments with overlaps. As shown in Figure 2, various primers, such as A105UF/A820 (820 bp), EVP4/A6141 (5594 bp), and A4879/A1005R (2488 bp), were used, as reported in Table 1. In addition, the PCR products were also visualized on an agarose gel to confirm that they were the correct size after amplification. The results revealed that this three-step method worked well for PCR assays.
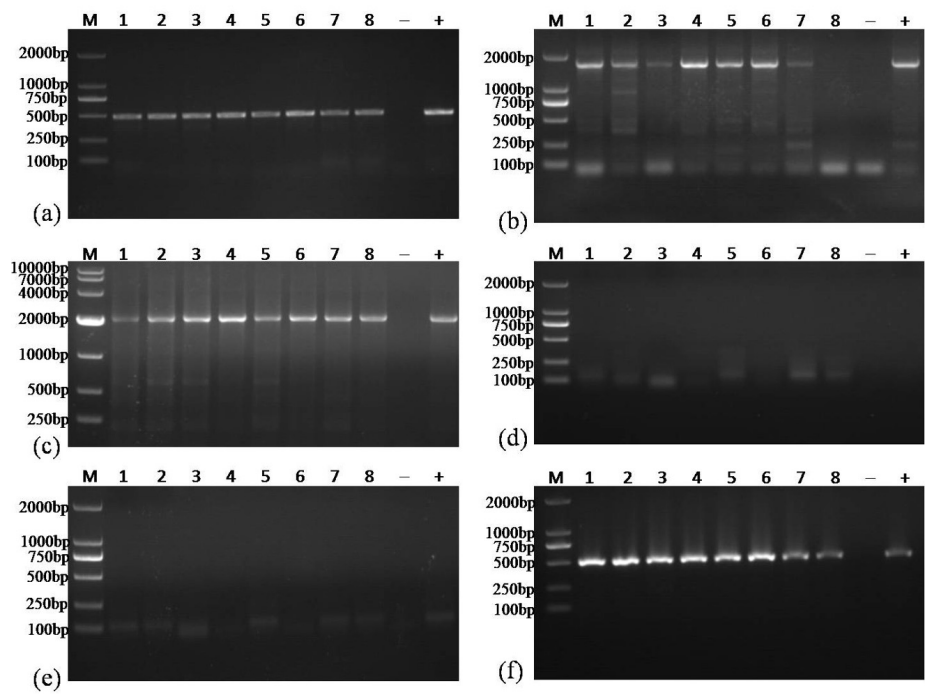

Figure 3. Agarose gel electrophoresis of the amplicons of A105UF/A105UR (a), A1001F/A1001R (b), A1002F/A1002R (c), A1003F/A1003R (d), A1004F/A1004R (e), and A1005F/A1005R (f) of eight different coxsackievirus A10 (CV-A10) strains. The FY01 to FY08 strains are represented in lanes 1 to 8 , respectively, and the negative and positive controls are labeled with "-" and "+". The DL2000 DNA marker was used in a, b, d, e, and f (TaKaRa, Dalian, China), and the DL10000 DNA marker (Takara, Dalian) was used in c.

\section{Optimization of PCR conditions}

To optimize the long fragment PCR conditions, the annealing temperature, annealing time, and extension time were rigorously tested based on the PrimeSTAR GXL DNA Polymerase manufacturer instructions. First, the annealing temperature of EVP4/A6141 was tested in the range $55^{\circ}-65^{\circ} \mathrm{C}$, and the most suitable temperature was found to be $63^{\circ} \mathrm{C}$. Second, the annealing time of EVP4/A6141 was examined from 0 to $30 \mathrm{~s}$, and the best time was found to be $15 \mathrm{~s}$. Third, the extension time of EVP4/A6141 was tested from 4 to $6 \mathrm{~min}$, and the shortest possible time was found to be $4 \mathrm{~min}$. Based on the PrimeSTAR GXL DNA Polymerase manufacturer instructions, the amount of cDNA for long fragment PCR should be between 250 and $750 \mathrm{ng}$. The viral suspension was concentrated by low-speed centrifugation according to the manufacturer instructions to produce the long fragment PCR product using EVP4/A6141.

\section{GenBank accession Nos.}

The near full-genome sequences of eight CV-A10 strains were BLASTed in GenBank, and the results revealed that they belonged to CV-A10 (GenBank accession No. KP009574-KP009581). 


\section{Whole-genome amplification using the six-step protocol}

To assess the six-step protocol, the primers reported by Hu et al. (2011) were used for the amplification of eight CV-A10 strains and the results obtained were compared with those obtained using the three-step protocol. As shown in Figure 3, A105UF/A105UR (a), A1001F/A1001R (b), A1002F/A1002R (c), A1003F/A1003R (d), A1004F/A1004R (e), and A1005F/A1005R (f) (Hu et al., 2011) were amplified under various conditions. The A1003F/A1003R and A1004F/A1004R primers resulted in a negative reaction. Therefore, it was necessary to develop a laboratory procedure for the full-length genome amplification of CV-A10 isolated from different countries and regions, and without any limitations.

\section{DISCUSSION AND CONCLUSION}

Currently, only two full-length genomes of CV-A10 have been reported (Oberste et al., 2004; Hu et al., 2011), probably because of the regional nature of its distribution or because it is underemphasized (Hu et al., 2011; Lu et al., 2012; He et al., 2013). Thus, there is very little information about the genetics and evolution of CV-A10. In previous studies, a method using universal primers was established to amplify the complete genome of CV-A10 (Oberste et al., 2004). In addition, a six-step protocol with specific primers was used for the amplification of the full genome of CV-A10 (Hu et al., 2011). As shown in Figure 3, the six-step protocol was used to obtain the complete genomes of eight CV-A10 strains. However, at least two of the PCRs ( $d$ and e) were unsuccessful. Moreover, nonspecific reactions ( $b$ and $c$ ) also took place.

This study presents a robust three-step protocol for the full-length genome amplification of CV-A10 based on long fragment PCR. Virus concentration and reverse transcription referred to the method for full-length genome amplification of a poliovirus strain (Chumakov, 1996; Boot et al., 2004; Laassri et al., 2005). The long fragment PCR was performed using EVP4/A6141 and the product was $5594 \mathrm{bp}$. As shown in Figure 2, a, b, and c produced fragments of sizes 750 to $1000 \mathrm{bp}, 4000$ to $7000 \mathrm{bp}$, and 2000 to $4000 \mathrm{bp}$, respectively. The results showed that the three PCRs carried out using the primers shown in Figure 1 were specific, accurate, and reproducible. Compared with the six-step protocol, the three-step protocol can not only effectively improve the specificity of the amplification and the range of applications, but also reduce the probability of errors in the full-genome amplification of CV-A10 using a step-by-step process. Furthermore, the three-step protocol not only simplifies the process of the whole-genome amplification of CV-A10, but also avoids cloning to the pGEM-T vector for sequencing. Thus, the universal primers method was not carried out as a comparison to the three-step method in this study.

A viral stock is a collection of different genomes that are closely related, and is often referred to as a quasispecies. A full-length RT-PCR on $\mathrm{CsCl}$-purified material of oral poliovirus vaccine poliovirus strains has shown that the quasispecies is preserved during the in vitro RNA-to-cDNAto-RNA conversion cycle (Chumakov, 1996; Boot et al., 2004). Like the poliovirus strains, CV-A10 is also a quasispecies. For this reason, we developed a three-step method that comprises one long fragment PCR, which is aimed at preserving the quasispecies nature of the viral stock as much as possible. A rapid DNA polymerase with high fidelity (PrimeSTAR GXL, see also the third subsection in the MATERIAL AND METHODS section) was used for long fragment PCR, because we hoped to achieve genome amplification that was quick and accurate. Thus, we expect that the three-step method will have a significant impact on the surveillance of the genetics and evolution of CV-A10. 


\section{Conflicts of interest}

The authors declare no conflict of interest.

\section{ACKNOWLEDGMENTS}

The authors would like to acknowledge all those who participated in this study. We thank the National Nature Science Foundation of China (\#81302701), the Grants for Scientific Research of BSKY (\#XJ201226) from Anhui Medical University, and the Natural Science Foundation of Anhui Province (\#1308085QC53) for financially supporting this study.

\section{REFERENCES}

Blomqvist S, Klemola P, Kaijalainen S, Paananen A, et al. (2010). Co-circulation of coxsackieviruses A6 and A10 in hand, foot and mouth disease outbreak in Finland. J. Clin. Virol. 48: 49-54. http://dx.doi.org/10.1016/j.jcv.2010.02.002

Boot HJ, Schepp RM, van Nunen FJ and Kimman TG (2004). Rapid RT-PCR amplification of full-length poliovirus genomes allows rapid discrimination between wild-type and recombinant vaccine-derived polioviruses. J. Virol. Methods 116: 3543. http://dx.doi.org/10.1016/j.jviromet.2003.10.008

Chang LY, Lin TY, Huang YC, Tsao KC, et al. (1999). Comparison of enterovirus 71 and coxsackie-virus A16 clinical illnesses during the Taiwan enterovirus epidemic, 1998. Pediatr. Infect. Dis. J. 18: 1092-1096. http://dx.doi.org/10.1097/00006454$199912000-00013$

Chumakov KM (1996). PCR engineering of viral quasispecies: a new method to preserve and manipulate genetic diversity of RNA virus populations. J. Virol. 70: 7331-7334.

Davia JL, Bel PH, Ninet VZ, Bracho MA, et al. (2011). Onychomadesis outbreak in Valencia, Spain associated with hand, foot, and mouth disease caused by enteroviruses. Pediatr. Dermatol. 28: 1-5. http://dx.doi.org/10.1111/j.15251470.2010.01161.x

He YQ, Chen L, Xu WB, Yang H, et al. (2013). Emergence, circulation, and spatiotemporal phylogenetic analysis of coxsackievirus a6- and coxsackievirus a10-associated hand, foot, and mouth disease infections from 2008 to 2012 in Shenzhen, China. J. Clin. Microbiol. 51: 3560-3566. http://dx.doi.org/10.1128/JCM.01231-13

Hu YF, Yang F, Du J, Dong J, et al. (2011). Complete genome analysis of coxsackievirus A2, A4, A5, and A10 strains isolated from hand, foot, and mouth disease patients in China revealing frequent recombination of human enterovirus $\mathrm{A}$. J. Clin. Microbiol. 49: 2426-2434. http://dx.doi.org/10.1128/JCM.00007-11

Ishiko H, Shimada Y, Yonaha M, Hashimoto O, et al. (2002). Molecular diagnosis of human enteroviruses by phylogeny-based classification by use of the VP4 sequence. J. Infect. Dis. 185: 744-754. http://dx.doi.org/10.1086/339298

Laassri M, Dragunsky E, Enterline J, Eremeeva T, et al. (2005). Genomic analysis of vaccine-derived poliovirus strains in stool specimens by combination of full-length PCR and oligonucleotide microarray hybridization. J. Clin. Microbiol. 43: 28862894. http://dx.doi.org/10.1128/JCM.43.6.2886-2894.2005

Lin TY, Twu SJ, Ho MS, Chang LY, et al. (2003). Enterovirus 71 outbreaks, Taiwan: occurrence and recognition. Emerg. Infect. Dis. 9: 291-293. http://dx.doi.org/10.3201/eid0903.020285

Lu QB, Zhang XA, Wo Y, Xu HM, et al. (2012). Circulation of Coxsackievirus A10 and A6 in hand-foot-mouth disease in China, 2009-2011. PLoS One 7: e52073. http://dx.doi.org/10.1371/journal.pone.0052073

McMinn PC (2002). An overview of the evolution of enterovirus 71 and its clinical and public health significance. FEMS Microbiol. Rev. 26: 91-107. http://dx.doi.org/10.1111/j.1574-6976.2002.tb00601.x

Mirand A, Henquell C, Archimbaud C, Ughetto S, et al. (2012). Outbreak of hand, foot and mouth disease/herpangina associated with coxsackievirus A6 and A10 infections in 2010, France: a large citywide, prospective observational study. Clin. Microbiol. Infect. 18: E110-E118. http://dx.doi.org/10.1111/j.1469-0691.2012.03789.x

Oberste MS, Peñaranda S, Maher K and Pallansch MA (2004). Complete genome sequences of all members of the species Human enterovirus A. J. Gen. Virol. 85: 1597-1607. http://dx.doi.org/10.1099/vir.0.79789-0

Osterback R, Vuorinen T, Linna M, Susi P, et al. (2009). Coxsackievirus A6 and hand, foot, and mouth disease, Finland. Emerg. Infect. Dis. 15: 1485-1488. http://dx.doi.org/10.3201/eid1509.090438

Pérez-Vélez CM, Anderson MS, Robinson CC, McFarland EJ, et al. (2007). Outbreak of neurologic enterovirus type 71 disease: a diagnostic challenge. Clin. Infect. Dis. 45: 950-957. http://dx.doi.org/10.1086/521895 
Shah VA, Chong CY, Chan KP, Ng W, et al. (2003). Clinical characteristics of an outbreak of hand, foot and mouth disease in Singapore. Ann. Acad. Med. Singapore 32: 381-387.

Wu Y, Yeo A, Phoon MC, Tan EL, et al. (2010). The largest outbreak of hand; foot and mouth disease in Singapore in 2008: the role of enterovirus 71 and coxsackievirus A strains. Int. J. Infect. Dis. 14: e1076-e1081. http://dx.doi.org/10.1016/j. iiid.2010.07.006

Yan JJ, Su IJ, Chen PF, Liu CC, et al. (2001). Complete genome analysis of enterovirus 71 isolated from an outbreak in Taiwan and rapid identification of enterovirus 71 and coxsackievirus A16 by RT-PCR. J. Med. Virol. 65: 331-339. http://dx.doi. org/10.1002/jmv.2038

Yu JH and Song HT (2010). Epidemiological analysis of hand, foot and mouth disease in Wendeng city in 2009. J. Prev. Med. Inf. 26: 649-650.

Zhang Y, Tan XJ, Wang HY, Yan DM, et al. (2009). An outbreak of hand, foot, and mouth disease associated with subgenotype C4 of human enterovirus 71 in Shandong, China. J. Clin. Virol. 44: 262-267. http://dx.doi.org/10.1016/j.jcv.2009.02.002

Zhang Y, Zhu Z, Yang W, Ren J, et al. (2010). An emerging recombinant human enterovirus 71 responsible for the 2008 outbreak of hand foot and mouth disease in Fuyang city of China. Virol. J. 7: 94. http://dx.doi.org/10.1186/1743-422X-7-94 